\title{
Decentralized Procurement Mechanisms for Efficient Logistics Services Mapping - a Design Science Research Approach
}

\author{
Tiphaine Henry \\ Orange Labs \\ Telecom SudParis, UMR 5157, \\ Institut Polytechnique de Paris, France \\ tiphaine.henry@orange.com
}

\author{
Roman Beck \\ IT University of Copenhagen, \\ Copenhagen, Denmark, \\ romb@itu.dk
}

\author{
Nassim Laga \\ Orange Labs \\ Paris, France \\ nassim.laga@orange.com
}

\author{
Walid Gaaloul \\ Telecom SudParis, UMR 5157, \\ Institut Polytechnique de Paris, \\ walid.gaaloul@it-sudparis.eu
}

\author{
Shenle Pan \\ MINES ParisTech, PSL University, \\ Centre de Gestion Scientifique, I3 \\ UMR CNRS 921760, Paris, France \\ shenle.pan@mines-paristech.fr
}

\begin{abstract}
Companies tend to outsource logistics services for flexibility or platform operating costs reduction. To do so, they typically use centralized platforms to delegate the services procurement process. However, those platforms can be prone to information asymmetries between carriers and shippers which can lead to sub-optimal procurement outcomes. A more transparent and efficient way to manage the procurement of logistics services between carriers and shippers could be a decentralized platform based on blockchain and smart contracts. In this paper, we design, implement, and evaluate the potential for a decentralized logistics services procurement system, following a design science research approach. In so doing, we contribute by (1) developing such a decentralized logistics services procurement system that addresses the allocation problem, and (2) developing a set of nascent design principles guiding the elaboration of decentralized procurement mechanisms on blockchain.
\end{abstract}

\section{Introduction}

Transport logistics is a complex process with many stakeholders involved that need to be coordinated to send a shipment from A to B. A central activity in that process is the freight transportation service procurement (FTSP) mapping, where shippers negotiate their transport needs with potential carriers. The FTSP activity challenge is to map the shipper delivery request with the best available carrier offer at a certain time and place (i.e., finding a match and issuing a contractual binding). Shippers can use dedicated platforms to find on-demand carriers to deliver merchandise [1]. For logistics services, the right mapping at the needed quality level is crucial, as the delivery quality impacts directly customer satisfaction and retention.

In this paper, we define problem owners as logistic companies involved in procurement mechanisms. The problem is worth exploring as shippers oftentimes struggle to find qualified carriers as information asymmetry in procurement marketplaces is strong $[2,3]$. Indeed, existing procurement marketplaces frequently lack information transparency: for example, access to delivery histories, or allocation protocol can be restricted. As a consequence, data integrity of the carrier profiles may not be assured, or important services records may not be kept [2]. Moreover, the freight procurement platform controls all information flows which gives it a lot of centralized power and an incentive to keep the mapping of logistics services nontransparent for others [3]. A procurement platform provider may feel incentivized to abuse their power to keep things nontransparent. One example is the so-called opaque (pricing) business model, whereby companies or platforms hide the (low) price of products to generate higher revenue (e.g., Priceline). This situation creates yet another problem, as the quality of services $(\mathrm{QoS})$ provided by carriers is not accessible freely by shippers. The lack of transparency of service allocation, influenced by information asymmetries, may lead to poor customer satisfaction. A study conducted among 300 retailers across the UK, US, and Germany in 2018 demonstrates that the average yearly cost of failed deliveries per retailer is almost $£ 200,000$ [4]. Thus, FTSP mapping requires a technology that (1) leverages 
trusted delivery information to compute the QoS of carriers, (2) fosters power balance between partners, and (3) optimize the FTSP process.

The blockchain has emerged in the past years as a new tool for trustworthy decentralized data storage and decision making $[5,6]$ with a potential for lower operational and transaction costs [7]. The blockchain ledger stores transactions in a tamper-proof fashion and smart contracts execute predefined protocols autonomously. Moreover, oracles can feed the blockchain with trustworthy external data: the blockchain connects to a number of oracles to triangulate the status of transaction outside the blockchain system.

A blockchain-based decentralized logistics services procurement marketplaces thus appears as an adequate technology to provide an efficient FTSP protocol with smart contracts securing the mapping and settlement of a logistics service in an efficient, finite, and autonomous way [8, 9, 7]. For accounting and compliance, such a system can even record all settled transactions in a tamper-resistant fashion, and keep track of delivery information provided by different oracles and tracking sensors. Such a decentralized procurement solution executed by smart contracts would improve the carrier-shipper mapping decision-making as well as the QoS. More precisely, the decision-making regarding the choice of the carrier would be improved as the protocol would be transparent and based on tamper-proof QoS data whose computation is agreed upon by all partners. The decision-making would be based on objective data and improve customer satisfaction on the long run. Moreover, the decision making would be beneficial for the carrier as her profile is put on equal footing with respect to other carriers.

Despite several publications of blockchain-based allocation protocols to map logistics services and their procurement, there is a research gap when it comes to incorporating information flows from oracles and tracking sensors. Thus, in our research, we focus on the efficient mapping of logistics services on a decentralized platform where information about QoS is available and incorporated into the procurement of logistics services and their mapping between shippers and carriers. Particularly, in this research we answer the following research questions: (RQ1) How can blockchain-based FTSP mapping assist shippers in the procurement of a logistics service with regards to process effectiveness, information transparency, and power balance between platform providers and platform actors? (RQ2) Which design principles need to be followed that guide the development of decentralized procurement mechanisms?
We investigate these questions by following a design science research approach anchored in the logistics field. We propose a blockchain-based FTSP mapping adapted to logistics to enhance the sector's needs for service flexibility and information transparency. The main contributions of this work comprise the list of the main requirements related to FTSP and derive a priori principles and features to match the procurement needs, as well as the design of an artifact holding the features that validates our approach through two evaluation cycles. Additionally, we contribute theoretically to the FTSP literature on blockchain use. To do so, we propose a set of design principles for the elaboration of decentralized FTSP, adapted to shippers needs. The three design principles are understandability of the mapping process by shippers, FTSP automation, and sensitive mapping metrics privacy.

The remainder of this paper is organized as follows. Section 2 introduces key concepts around blockchain-based FTSP mappings. Section 3 reviews related work. Then, section 4 presents the design science research methodology approach. In section 5, we describe the blockchain-based FTSP mapping design requirements and define the design features needed for blockchain-based FTSP mapping. We present our practical and academic contributions derived from the iterative design cycles in section 6 . The paper concludes with section 7 .

\section{Preliminaries}

This section introduces some key concepts about FTSP and the blockchain technology, before investigating related work on decentralized allocation protocols using blockchain.

The freight transportation procurement process is one of the primary activities in the logistics field. The FTSP holds three stakeholders: the shipper (i.e., the service buyer) who initiates the allocation request, the carrier (i.e., the service seller) who sells its delivery services, and an intermediary, often a digital platform, responsible for the carrier-shipper allocation [10, 11]. The allocation request concerns two market types: long-term markets, which are delivery contracts signed for years, for example, and spot markets concerning one-shot agreements for a single specific request. It comprises two stages: first, the matching stage, where the set [service request/resource provider] is defined, and then the allocation, which generates a mediation contract, the CMR (i.e., CMR Convention: Convention on the Contract for the International Carriage of Goods by Road). In a coopetition context [12, 13], both carriers and shippers are looking to cooperate for cost 
optimization and efficiency gains [10, 14]. Especially, carriers can also use the platforms to exchange on-hand merchandise. Indeed, platforms optimize transportation costs and enact allocations autonomously. Multi-modal freight transportation among carriers is a practical example. It is worth noting that the platforms' service procurement process will be the same for either shipper-to-carrier or carrier-to-carrier allocations.

A blockchain is a distributed database based on a peer-to-peer network, i.e., a database spread across multiple nodes, or servers [15]. Blockchains keep track of the transfer of assets between several parties. They lower the cost of trusted transactions by making databases tamper-proof by design [16]. Blockchain systems store data transactions hierarchically, using linked blocks of aggregated transactions [5]. Cryptography hashes and asymmetric encryption enforce the non-tampering of the transactions. The trusted behavior of blockchain systems builds upon the consensus protocol used to update the chain of blocks. The consensus protocol states the strategy used to settle on a shared state of truth. This protocol ensures the tamper-proof growth of the database [5]. Smart contracts are deterministic scripts enforcing a set of agreements between parties in the blockchain [17]. Smart contracts can use oracles to connect to off-chain data, coming from sensors or databases [18].

Design science research refers to a scientific method to develop artifacts answering real-world problems [19, 20]. Its interest lies into the methodology making sure that the identification and specification of the underlying problem are realized, leading to its resolution at some level of completeness and abstraction. Consequently, stakeholders and their problem are considered before designing a solution. The finality of a design science research approach is to abstract design knowledge to generate theoretical insights framing the building of future solutions. A design science research approach comprises the three main steps. The first step refers to the analysis of the problem leading to generating a set of objectives. A set of features are then derived from these objectives. The second step is to develop and evaluate the artifact iteratively. The third step is to abstract design knowledge under a set of design principles.

\section{Related-work}

Compared to traditional allocation systems, blockchain-based allocation protocols unlock trustworthy process automation $[9,21,22]$. Indeed, integrity of the protocol can be ensured as all carriers are considered before attributing a request, while historic data stored in the blockchain is tamper-proof.
When a conflict occurs, the history of transactions can be retrieved and used as the single source of truth. Blockchain-based FTSP mappings can moreover go one step further by managing autonomously and reliably the end-to-end service enactment.

Table 1 presents the main known related work investigating blockchain support for allocation procedures. On the one hand, several papers investigate the use of blockchain and smart contracts for the autonomous allocation of services such as energy or computation power $[23,24,25,2,26]$. These papers leverage smart contracts for autonomous contractualization $[24,2]$, incentives to encourage service completion [25, 2], or delivery settlement [27]. However, these papers do not focus specifically on the FTSP use case, and do not use oracles to compute QoS ratings or integrate sensor feedbacks. Moreover, these artifacts were not realized using a design science research approach and thus do not provide design principles for developing decentralized procurement mechanisms on blockchain. On the other hand, several papers propose a design science research approach to investigate the use of blockchain applications. Two papers, [28, 29], focus respectively on the development of blockchain-based IoT applications and smart-parking. However, the retrieved design principles do not concern FTSP mappings specifically, and oracles are not integrated in the research works. Two design science research papers, [30,31], focus on the use of blockchain for logistics but do not focus specifically on the FTSP protocol. The first paper focuses on the management of bills of lading via blockchain. Retrieved design principles are process digitization, tamper-proof storage, accessibility, and user authentication. The second paper focuses on food supply chains; the retrieved design principles are mainly related to data privacy and keeping sensitive data off-chain. It is also to note that none of the retrieved work leverages oracles to realize a QoS-based allocation.

Thus, in this research, we address this research gap regarding the potential of decentralized FTSP mapping using blockchain, smart contracts, and oracles. We do so using a design science research approach.

\section{Design science research methodology}

We follow a design science research approach to elaborate a blockchain-based FTSP mapping (cf. section 2) answering the needs of shippers striving to find the most qualified delivery drivers in open and dynamic markets. Our rationale for choosing the design science research approach is the scarcity of (1) decentralized procurement mechanisms on blockchain, 
Table 1. Blockchain artifacts in design science research (DSR)

\begin{tabular}{|c|c|c|c|c|}
\hline$\stackrel{4}{\mathscr{\imath}}$ & 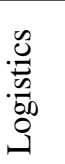 & 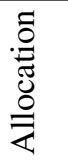 & $\begin{array}{l}\frac{\ddot{T}}{\tilde{O}} \\
\stackrel{0}{0}\end{array}$ & $\frac{\alpha}{\mathscr{D}}$ \\
\hline$[23,24,25,2,26,27]$ & no & yes & no & no \\
\hline$[28,29]$ & no & no & no & yes \\
\hline$[30,31]$ & yes & no & no & yes \\
\hline Our approach & yes & yes & yes & yes \\
\hline
\end{tabular}

and (2) FTSP field evaluations in the blockchain literature. In road transport fully digital CMR (e-CMR) is far from standard recognized by e.g. custom authorities in many regions/countries. In the experiments, we make the assumption that e-CMR digitization is accepted and the norm.

First, we analyze the literature regarding FTSP to analyze the problem, propose a list of design requirements to answer FTSP-related issues, merge it into a set of three design objectives, and decline each objective into a set of features. Next, we start the prototype development. We proceed with conducting two iterative development/evaluation cycles.

During the first iteration, we evaluate the artifact through user testing. We proceed to a moderated usability test of the prototype. Our goal was to assess the prototype with the maximum and diverse problem owner representatives. We proceeded to the tests with master students of the Service-oriented architecture field. to palliate the difficulty to experiment with the logistics industry. We tested students as they were close to shippers, i.e., blockchain agnostic. We conducted the experiments with the maximum number of participants to have a diversity of answers, we obtained 14 answers. During experiments, students were asked to act as independent shippers. They followed provided instructions to access the dApp. Afterwards, they followed a scenario asking them to find a delivery person fitting a set of requirements. Afterwards, a questionnaire was given to participants comprising questions related to their overall feedback, the dApp industrial applicability, and usability flaws.

The second iteration of the artifact involved two focus groups. The groups were composed respectively of two academics in freight transportation and three industry experts specialized in the procurement process of logistics services. The choice of interviewing logistics specialists was motivated by their direct relation with logistic stakeholders. They hence had a broad understanding of the variety of issues related to shippers. Additionally, they were involved in ongoing projects on digitization of logistic networks in France, and aware of specific challenges related to blockchain. Each session followed the same methodology. We launched a demonstration of the artifact before letting the experts debate. The focus groups lasted around one hour each. We did not launch a naturalistic evaluation in a freight procurement setting as the artifact is presented as a minimum viable product, designed to understand how the blockchain can improve current FTSP mechanisms (cf. [32]).

Finally, we focus on knowledge abstraction and theory generation. We extracted overlapping semantics from the testimonies to identify nascent design principles. We hence propose three nascent design principles based on our iterative empirical development and evaluation cycles. Besides, we confront the proposed nascent design principles under the light of nascent design principles extracted from other design science research papers.

\section{Designing a blockchain-based FTSP mapping: from requirements to design principles and features}

\subsection{Designing requirements}

To get the list of design requirements linked to enhanced FTSP platforms, we carry out a set of interviews with academics in freight transportation. We also query the literature to gather articles related to (1) FTSP mapping current issues, and (2) blockchain-based FTSP mapping proofs-of-concept. More precisely, we generate two subjective search strings [33]. The first one refers to current FTSP mapping limitations The output search string is ("freight transportation" OR "service procurement" OR "freight allocation" OR logistics) AND (challenges OR issues). The second search string refers to resource allocation and blockchain. The output search string is (resource allocation OR market place OR matching OR freight procurement) AND (blockchain OR DLT OR smart contracts).

We identify five design requirements that a resource allocation artifact should meet to circumvent current FTSP mapping issues (R1-R5 hereinafter).

First, a solution must provide allocation flexibility (R1) to shippers and carriers [10, 2, 34]. On the shipper side, the shipment flows are variable and costly, primarily via spot markets. On the carrier side, there is a need to exchange and re-bundle items effortlessly with competitors to perform economies of scale (e.g., increasing fill rate) and economies of scope (e.g., lane exchanges). Second, a solution must provide an autonomous allocation process (R2) [35, 36, 37]. The 
flexibility is low, and frequently trucks are half-full for spot deliveries [38]. One of the causes is the heavy load of administrative tasks, with still paper-based contracts, e.g., the $C M R$ papers [37]. The third requirement is that a solution must provide enact real-time payment (R3) to help carriers optimize their commercial processes and operating costs $[10,39,37,40]$. There is a need for a cost-efficient, secure, and fast payment process right after delivery on the carrier side [38]. Fourth, a solution must comply with CMR regulations (R4) [41, 42, 39]. Indeed, the administrative overwork linked to CMRs can discourage flexible hiring and item re-bundling. Finally, a solution must provide delivery history integrity and traceability (R5) [43, 44, 37, 45, 34, 40]. The allocation should be objective and delivery history should be traceable. Additionally, carriers and shippers should not be able to modify or contest a settled delivery.

\subsection{Artifact principles}

We gather the aforementioned (R1)-(R5) requirements into three principles (i.e., P1, P2, P3 hereinafter) that a resource allocation artifact should meet to circumvent current FTSP issues.

First, platform operating costs reduction (P1) of the FTSP system should be considered to enhance the transportation process. On the one hand, platform operating costs reduction relies on an eased allocation process (cf R2). Fostering autonomous freight procurement systems would render the procurement infrastructure less costly for each stakeholder as they would need to provide less time to administrative activities (e.g., settling a delivery). On the other hand, platform operating costs reduction also relies on allocation flexibility (cf R1). Indeed, customized allocation requests will be more prone to answer the shipper's standards. Consequently, less time will be spent to finding the perfect carrier for a task, and the risk for failed deliveries due to poor customer satisfaction will decrease. Blockchain smart contracts can answer this need for cost reduction, as they can be used as autonomous services in untrusted environments [30].

Second, the platform should offer contractual flexibility (P2). The inner flexibility of the allocation mechanism ensures contractual flexibility (cf R1). For example, if the allocation mechanism makes it possible for freight re-bundling, it will enhance contractual flexibility. Contractual flexibility is also related to real-time payment (cf R3). The fast, simple and secure payment processing enabled by the smart contracts will make carriers be paid right after delivery to accelerate the cash flow and flexibility. Real-time payment and contractual flexibility can be managed by a dedicated smart contract. The latter could manage offers, and re-bundle them if needed.

Finally, allocation integrity (P3) should be considered, be it for the allocation process, the record of service rates, and the management of CMR. The integrity of the digitalized CMR (cf R4) is paramount for regulatory authorities. Integrity in terms of delivery history and protocol should be considered (cf R5). Providing adequate performance feedbacks to future shippers requires delivery history integrity. Meanwhile, an objective allocation requires protocol integrity: the protocol should consider all carriers for a given service request without bias. The integrity of CMRs and delivery history can be ensured by the tamper-proof affordance of the blockchain ledger [30]: all approved transactions cannot be contested nor changed. The integrity of the allocation is moreover ensured by smart contracts as they are deterministic: the inner logic of the smart contract will be respected at each stage of the allocation process.

\subsection{Emerging features}

We elaborate a set of blockchain-based FTSP mapping features to answer the needs for platform operating costs reduction (P1), contractual flexibility (P2), and allocation integrity (P3) in FTSP patterns (see section 6.1 for implementation details).

Regarding platform operating costs reduction (P1), we propose an autonomous allocation mechanism to reduce cognitive weights on the user side, coupled to the generation of a digital CMR agreement. Moreover, we offer to integrate real-time payment facilities once the delivery completes. On the shipper side, there are minimum search actions to find a carrier and enact an agreement. On the carrier side, the allocation is faster and requires less paperwork: the overall allocation cost decreases. Regarding contractual flexibility (P2), we propose a customizable QoS allocation to the shipper. The latter can tune each rating's weights referred to as $P_{O}$ to compute the carriers' QoS. The QoS is calculated as a weighted normalized mean, using both $P_{O}$ and the set of weighting parameters $\alpha: \forall i \in$ Candidates, $Q o S_{i}=\sum_{j=0}^{n} \alpha_{j}\left\|P_{O_{j}}\right\|$. If a weighting factor equals zero, then $P_{O}$ is not considered for the QoS computation. If it equals one, then $P_{O}$ will be fully considered for the QoS computation. For example, if the carrier experience is a primary criterion of selection, the shipper can set a weight of 1 to this parameter. Finally, concerning allocation integrity (P3), the blockchain can map carrier offers (e.g., pricing or availability) to their tamper-proof records of services, accessible via a dedicated profile smart contract recording the QoS of 
each service. Moreover, a smart contract operates the allocation to ensure protocol integrity and objectivity: the carrier with the highest QoS (or the lowest costs or $\mathrm{CO} 2$ emissions, etc.) will obtain the assignment.

Tamper-proof performance feedbacks can have multiple shapes, e.g., delivery time, temperature threshold, or customer satisfaction ratings. The combination of both subjective (customer satisfaction rating), and objective metrics (temperature threshold or delivery time) constitute the QoS and thus moderate poor performance reviews. Moreover, the QoS update can be computed with a weighted exponential average that puts more weights on latest deliveries. Additionally, at initial stages, new comers are assigned with a maximum QoS rating to avoid the case where they would never be selected.

The following section presents the findings generated from the building of an artifact holding these features.

\section{Implementing a decentralized trust-free FTSP mechanism}

In this section, we present the practical contribution of this research work: the decentralized FTSP prototype. We then focus on the academic contribution by discussing the nascent design principles emerging from the development and evaluation cycles.

\subsection{The blockchain-based FTSP mapping prototype}

We propose a blockchain-based FTSP mapping protocol to fulfil the needs for platform operating costs reduction, allocation flexibility, and allocation integrity. The prototype architecture is multi-tiers, with (i) an application layer, (ii) the backend (smart contract, oracle) running on a blockchain, (iii) a Rest API for bridging the application layer with blockchain.

The blockchain-based FTSP mapping process follows the subsequent steps (cf. Fig. 1). Initially, representatives from both shippers and carriers instantiate the FTSP mapping. The representatives state the metrics that the smart contract will use to compute the on-chain QoS. Afterward, the carrier-shipper mapping can occur. Two stakeholders, carriers and shippers, interact with the FTSP smart contract. Carriers share with the smart contract eligibility information (e.g., location, licenses, truck capacity, and equipment) (step 1.a.). The smart contract associates this information with initially empty QoS metrics used to record the history of their past services (e.g., number of deliveries, and average delay) (step 1.b.). Shippers trigger the smart contract to find a carrier matching their needs by specifying filtering criteria (e.g., price, location, shipping date, equipment, merchandise volume) and sorting criteria (e.g., preferred experience, or maximum delay) (step 2.). The smart contract will filter the candidates (step 3.a.) and delegate the QoS computations according to the required sorting criteria to the oracle (step 3.b.-3.c.). The oracle provides to the smart contract the best-matching carrier (step 3.d.). Finally, the smart contract generates a digital delivery agreement as a decentralized version of the CMR (step 4.). The user (shipper or carrier) can retrieve all contracts bound to its public address.

The need for an oracle originates from the need to carry on QoS computations with past services history stored on the ledger. Such computation cannot be carried on using a smart contract due to gas limits imposed by blockchain architectures such as Ethereum. Thus, an external trusted entity is used to carry out computation: the oracle triangulates the results coming from a trusted API query to avoid any information tampering risk. Regarding the architecture of the prototype, oracles exchange information with the blockchain, and end-users exchange information with the blockchain. Hence there is no more intervention of a trusted third party.

We refined the prototype following two iterative cycles. User tests underlined the need to display computation information to enhance trust in the system. Indeed the black-box effect appeared, linked to users using a technology they are not expert with occurs. Users can only trust the machine, as they do not know the underlying protocols. This dependency may trigger mistrust. We thus displayed all resource profiles to the users and the ratings obtained for each resource. The second evaluation round consisted of focus group discussions. Testimonies underlined the need for an almost entirely autonomous system. We revisited the artifact by merging the matching and allocation stages into one single step.

Power asymmetry between carriers and shippers is addressed through a shared governance which can take place through an open or consortium blockchain: each actor participates equally to the consensus protocol. Information asymmetry is addressed by making accessible to shippers carriers' former services rating on the blockchain ledger. Additionally, power balance is reached by putting into escrow the carrier's pay, as well as the carrier's caution. Hence, both the shipper and the carrier are bound after validation of the mapping. The smart contract enables platform operating costs reduction by managing allocations autonomously. The smart contract also allows contractual flexibility as it speeds cash flow; payment 


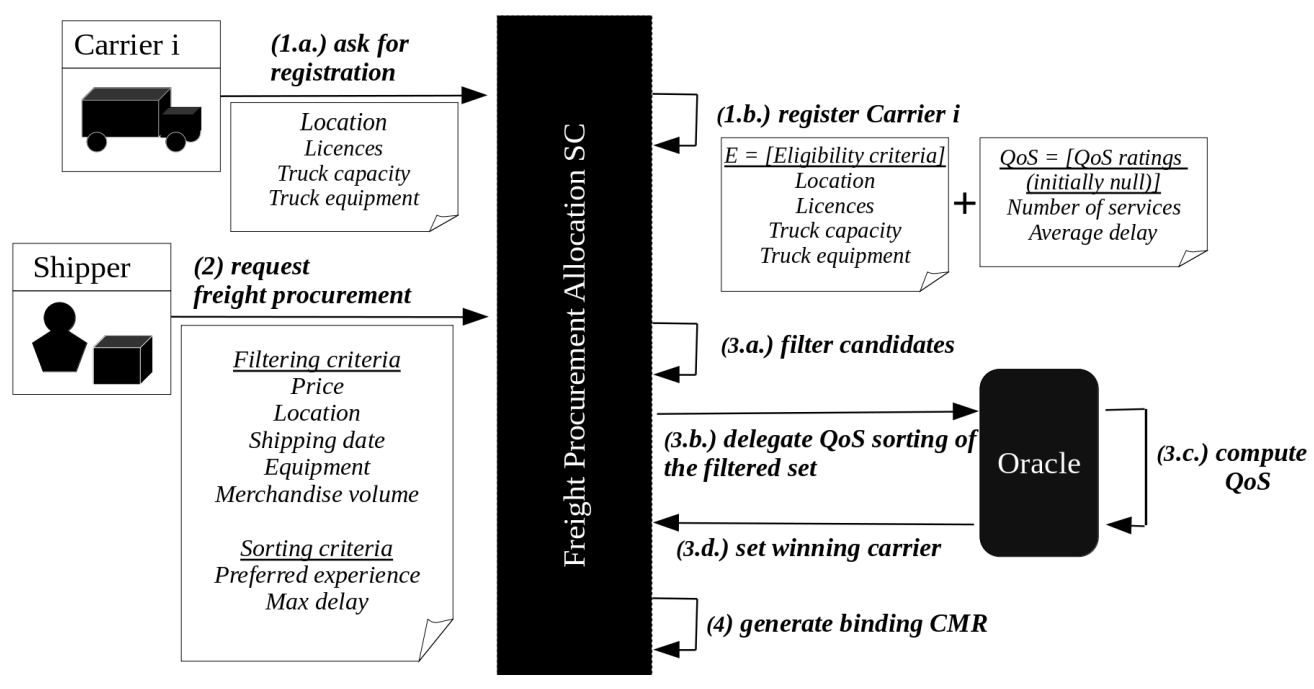

Figure 1. Blockchain-based FTSP mapping protocol

is enacted right after the shipping is settled. Finally, the combination of (1) the blockchain ledger, (2) the smart contract protocol, and (3) the oracle-based QoS computations ensures tamper-proof and objective management of the allocation requests. With the proposed architecture, oracles share QoS-computation results with the blockchain, and shippers and carriers interact directly with the blockchain to manage delivery requests. Hence, there is no more intervention of a trusted third party. Thus, carrier-shipper mappings can be done in a secure, trusted, reliable way by a blockchain system. As a side note, regarding carrier coopetition, a carrier wishing to delegate the delivery service to another carrier can take the role of the shipper. The generated CMR contracts holds a link to the initial CMR contract. When the new carrier-carrier CMR is settled (i.e., the delegated delivery has been fulfilled), the status of both CMR contracts is set as settled, and the smart contract transfers the corresponding escrowed payments. The QoS rating obtained for the delivery is stored in both carrier profiles.

\subsection{Theory generation}

In this subsection, we propose three nascent principles stemming from the prototype development cycles in order to dig onto the reasons making the artifact successful [46]. We then discuss these findings under the light of other design science research papers.

Table 2 presents the three emerging design principles detailed hereinafter. First, understandability of the smart contract shipper-carrier allocation is needed to encourage non-technical users adoption (DP1). Delegating the allocation process to the blockchain
Table 2. Blockchain-based FTSP mapping nascent design principles

\begin{tabular}{|ll|}
\hline $\begin{array}{l}\text { Understand- } \\
\text { ability }\end{array}$ & $\begin{array}{l}\text { The smart contract shipper-carrier } \\
\text { allocation protocol must be } \\
\text { displayed to the shipper }\end{array}$ \\
\hline Automation & $\begin{array}{l}\text { The FTSP process must be carried } \\
\text { on in an end-to-end fashion by the } \\
\text { smart contract }\end{array}$ \\
\hline & $\begin{array}{l}\text { The allocation mechanism must } \\
\text { QoS-metrics } \\
\text { privacy }\end{array}$ \\
\hline
\end{tabular}

triggered some concerns during the user testing stage. More precisely, the use of an oracle raised concerns related to integrity gains. Having no access to the decision protocol, several users were puzzled regarding the end result, as they did not have access to the smart contract reasoning for such allocation. Thus the FTSP platform should display the decision protocol alongside the data output to avoid the technological black-box effect. The blockchain architecture indeed requires strong technical skills to understand its benefits (decentralized governance, tamper-proof data, autonomous scripts). For non-expert users, the prototype thus consists of a box where data comes in (e.g., their delivery request), and data comes out (e.g., a shipper/carrier mapping), without any understanding of what happens inside the box. We propose to display to the shipper computation outputs for each profile to counter the black-box issue. Moreover, automation of the FTSP process is necessary (DP2): the FTSP process must be carried on in an end-to-end 
fashion by the smart contract. Indeed, one of the significant benefits underlined by both testers and experts is process optimization. Experts have made suggestions for enriched process automation. More precisely, they foresee blockchain-based FTSP mapping as autonomous agents managing request bundling, re-pricing, or matching. To integrate automation into our artifact, we merge the service request and contractualization stages into one step. The service status is tracked and managed within the blockchain for integrity and efficiency. Nonetheless, oracle latency is at stake regarding allocation queries, as it can take up to several minutes to process an request. According to one expert, 'latency variability seems acceptable for long-term markets where contracts can be set for a year or so [...] In spot markets, the service provider could become unavailable before the end of the query.' Thus, the automation of the FTSP mapping should take into account allocation latency. Finally, metrics privacy should be considered (DP3). The allocation mechanism must keep sensitive data off-chain in competitive markets. On the evaluation side, the disjunction between private and public data appears in several testimonies. Such sensitivity needs to adjust to the market at stake. Price and capacity data are sensitive and should remain private to other carriers' eyes. Though our prototype saves QoS metrics to the blockchain, a better option is to encrypt the price and volume metrics. We leave it for future work.

These nascent design principles resonate with several design principles proposed in the design science research literature. Accessibility of blockchain-based applications to non-technical users is one of the design principles of [30]. The design principle (DP1) stands on the necessity to explain adequately the smart contract decision-making process to users. Though blockchain smart contracts prone automation and transparency, protocol and data trust comes at the condition that users understand well the mechanisms motivating a decision (a delivery request allocation here). (DP1) is thus reinforced as a design principle. Moreover, allocation automation (DP2) resonates with the need for digitizing paper-based processes in [30]. The issuance of CMR contracts implies the production of several paperwork copies that need to be approved by both shipper and carrier at the start of the delivery. Consequently, the paperwork slows down the delivery process, reduces overall efficiency, and implies tampering risks. (DP2) also resonates with the potential for smart contracts to manage processes presented in [29]. (DP2) is thus also reinforced as a design principle. Lastly, the privacy of allocation metrics (DP3) resonates with the need to manage in an off-chain fashion private and sensitive data. These needs were underlined in [47] that performed a design science research study in the trucking industry: private carrier and shipper information should remain off-chain. The need for sensitive data privacy is also underlined as a design principle in [31] and [28]. QoS metrics act as private and sensitive data in our setting. In the future, the QoS metrics of our proposed mechanism should consequently whether be ciphered so that they cannot be understandable by other competitors, or kept off-chain. Thus, (DP1-3) can be considered as design principles for the development of a blockchain-based FTSP solution.

The contribution of this paper to theory lies into investigating the use of the blockchain in the logistics industry with respects to coopetition [12]. The current FTSP related literature mostly focuses on the traditional carrier-to-shipper market. Little attention has been paid to carrier-to-carrier market; and the application of blockchain and smart contract in such market is still a brand new research topic. The FTSP process is applied in a cooperative and coopetive environnement as (1) competing carriers agree to use the same platform to gain a service auction, and (2) competing shippers agree to use the same platform to find an adequate carrier. Both shipper and carrier participants have a shared interest in using the blockchain-based FTSP platform as the system has the potential to reduce operational costs, increase visibility of the carriers, and transparency and integrity of the deliveries.

\section{Discussion, conclusion, and future work}

This paper focuses on answering the need of logistics services procurement for more effectiveness of the carrier-shipper mapping process, alongside information transparency, and power balance. To do so, we investigate on the potential of the blockchain technology that offers decentralized and trustworthy scripting capabilities. To this aim, we carry out a design science research project focusing on the use of blockchain for decentralized logistics services procurement.

The paper addresses two research gaps in the FTSP literature on blockchain use: the scarcity of blockchain-based freight procurement applications, and the scarcity of theory-driven considerations. To address these concerns, we present a prototype that illustrates how blockchain can help to make carrier-shipper allocation processes more autonomous, objective, and trustworthy. The issue of information asymmetry is addressed as blockchain smart contracts remove the need for a trusted third party. The tamper-proof capability of the blockchain ledger answers the need 
for information transparency. Finally, the use of smart contracts also fosters process effectiveness as qualitative mappings can be reached through leveraging blockchain-based carrier QoS. We also provide nascent design principles for FTSP applications that aim to mitigate the transactional risk and uncertainty that is inherent to logistics systems. More precisely, we derive three design principles for the design of blockchain-based FTSP mappings based on the study: understandability, automation, and metrics privacy. The need for understandability of the mapping process precises the will for more information transparency in mapping systems: the access to information must be linked to an imperative of understanding. The process automation design principle may answer the need for power balance and flexibility: the more autonomous the process, whose steps are agreed upon by a consortium of shippers and carriers, the more balanced the overall process. The need for metrics privacy may however be controversial, as users wish both information transparency and privacy. A compromise may be reached with cryptography protocols such as homomorphic encryption to leverage carriers' QoS without revealing sensitive information.

The practical applicability of the prototype may be prone to reluctance due to the difficult digitization of the logistics industry. The use of decentralized applications (dApps) may ease the appropriation of the proposed system. The dApp can be used by SME users as a normal app on a smartphone, which connects to the blockchain. Additionally, smart contracts will automatize some operations such as billing, hence the system could fasten the digitization of these companies. Interoperability between mapping apps currently generates many tensions today. Blockchain, or cross-chain technologies could address this practical issue. In practice, a user could use a mapping dApp, and provide its data to gain mapping services in a secure and fast fashion. Furthermore, to fit current logistics processes, not yet fully digitized, and using a payment term additionally to the CMR, the notion of real-time payment could be extended with a digitized payment term. This payment term would be managed through a dedicated smart contract, instantiated by the mapping smart-contract after the e-CMR generation.

Several limitations appear in this prototype. First, smart contracts use oracles to aggregate blockchain-available QoS data using an API. Issues related to API tampering may occur. The oracle could call independent computation APIs and compare the results to ensure results integrity. Additionally, a party wishing to claim a computation failure may use blockchain data to verify computations. What is more, we use a public blockchain to demonstrate the feasibility of the solution, which may lead to an inadequate balance of powers. To ensure an adequate balance of powers between shippers and carriers, a future solution would require a permissioned blockchain dedicated to the mapping process, whose smart contracts are generated by a consortium of shippers and carriers. To be close to the field's reality, we designed the artifact by extracting the main allocation metrics used in FTSP. For the user tests, we gathered a population with no experience of blockchain. Thus we can assume that their answers are close to carriers discovering the technology for the first time. Furthermore, the focus groups comprises logistics field experts: we can assume that we qualitatively assessed the potential of blockchain-based FTSP mapping in the logistics context. Regarding internal validity and results correctness, we tried to influence the least possible user testing experiments by letting the users free to use the application. The focus group panel was also broad enough to underline the most salient benefits and challenges of using blockchain-based FTSP mappings. However, we acknowledge that the logistics researchers and the industry experts are an estimator of what happens in logistics services' procurement process. At last, the design science research approach is anchored in a FTSP context, and thus findings are not generalizable.

An avenue for future work is to leverage our platform to answer the metrics privacy design principle. To do so, we plan to investigate on the use of cryptography methods to cipher the data to be computed for the QoS in order to preserve procurement privacy.

\section{References}

[1] W. Zhu and S. C. Ng et al, "The role of outsourcing management process in improving the effectiveness of logistics outsourcing," Int. J. Prod. Econ., 2017.

[2] M. Nardini, S. Helmer, N. El Ioini, and C. Pahl, "A blockchain-based decentralized electronic marketplace for computing resources," SN Computer Science, 2020.

[3] M. Van Deventer, C. Brewster, and M. Everts, "Governance and business models of blockchain technologies and networks," tech. rep., TNO, 2017.

[4] Loqate, "Fixing failed deliveries, stamping out faulty fulfilment," 2018.

[5] G. Wood et al., "Ethereum: A secure decentralised generalised transaction ledger," Ethereum project yellow paper, vol. 151, no. 2014, pp. 1-32, 2014.

[6] J. Mendling, I. Weber, et al., "Blockchains for business process management-challenges and opportunities," TMIS, 2018.

[7] S. Pan, D. Trentesaux, D. McFarlane, B. Montreuil, E. Ballot, and G. Q. Huang, "Digital interoperability in logistics and supply chain management: state-of-the-art and research avenues towards physical internet," Computers in Industry, vol. 128, p. 103435, 2021. 
[8] S. Oranburg and L. Palagashvili, "The gig economy, smart contracts, and disruption of traditional work arrangements," Smart Contracts, and Disruption of Traditional Work Arrangements, 2018.

[9] D. Röck, "The foundation of distributed ledger technology for supply chain management," in HICSS, 2020.

[10] M. Lafkihi, S. Pan, and E. Ballot, "Freight transportation service procurement: A literature review and future research opportunities in omnichannel e-commerce," TRANSPORT RES E-LOG, 2019.

[11] C. Weinhardt and H. Gimpel, "Market engineering: An interdisciplinary research challenge," in Dagstuhl seminar proceedings, 2007.

[12] M. Bengtsson, J. Eriksson, and J. Wincent, "Coopetition: new ideas for a new paradigm," Coopetition: Winning strategies for the 21st century, pp. 19-39, 2010.

[13] H. Shvindina, "Coopetition as an emerging trend in research: perspectives for safety \& security," Safety, vol. 5 , no. 3, p. 61, 2019.

[14] C. Limoubpratum, H. Shee, and K. Ahsan, "Sustainable distribution through coopetition strategy," Int. J. Logist. Res. Appl., 2015.

[15] H. Wang et al., "Blockchain challenges and opportunities: a survey," IJWGS, 2018.

[16] J. Mendling et al., "Blockchains for business process management - challenges and opportunities," $A C M$ TMIS, 2018.

[17] K. Christidis and M. Devetsikiotis, "Blockchains and smart contracts for the internet of things," IEEE Access, vol. 4, pp. 2292-2303, 2016.

[18] H. Al-Breiki, M. H. U. Rehman, K. Salah, and D. Svetinovic, "Trustworthy blockchain oracles: review, comparison, and open research challenges," 2020.

[19] K. Peffers, T. Tuunanen, M. A. Rothenberger, and S. Chatterjee, "A design science research methodology for information systems research," JMIS, 2007.

[20] R. Beck, S. Weber, and R. W. Gregory, "Theory-generating design science research," Information Systems Frontiers, 2013.

[21] C. Sturm, J. Scalanczi, S. Schönig, and S. Jablonski, "A blockchain-based and resource-aware process execution engine," FGCS, 2019.

[22] O. López-Pintado, M. Dumas, L. García-Bañuelos, and I. Weber, "Controlled flexibility in blockchain-based collaborative business processes," IS, 2020.

[23] S. Wang et al., "Permissioned blockchain for efficient and secure resource sharing in vehicular edge computing," arXiv, 2019.

[24] M. Troncia, M. Galici, and M. Mureddu et al, "Distributed ledger technologies for peer-to-peer local markets in distribution networks," Energies, 2019.

[25] Z. Li, J. Kang, R. Yu, D. Ye, Q. Deng, and Y. Zhang, "Consortium blockchain for secure energy trading in industrial internet of things," IEEE TII, 2017.

[26] S. Myung and J.-H. Lee, "Ethereum smart contract-based automated power trading algorithm in a microgrid environment," The Journal of Supercomputing, 2020.

[27] A. Pinna and S. Ibba, "A blockchain-based decentralized system for proper handling of temporary employment contracts," in SI conference, Springer, 2018.
[28] J. Lockl, V. Schlatt, A. Schweizer, N. Urbach, and N. Harth, "Toward trust in internet of things ecosystems: Design principles for blockchain-based iot applications," IEEE Transactions on Engineering Management, 2020.

[29] S. Ahmed, M. S. Rahman, M. S. Rahaman, et al., "A blockchain-based architecture for integrated smart parking systems," in 2019 PerCom Workshops, 2019.

[30] K. Nærland, C. Müller-Bloch, R. Beck, and S. Palmund, "Blockchain to rule the waves-nascent design principles for reducing risk and uncertainty in decentralized environments.," in ICIS, 2017.

[31] R. Sharma, S. Wingreen, N. Kshetri, and T. Hewa, "Design principles for use cases of blockchain in food supply chains," in AMCIS, 2019.

[32] J. Venable, J. Pries-Heje, and R. Baskerville, "Feds: a framework for evaluation in design science research," Eur. J. Inf. Syst, 2016.

[33] H. Zhang, M. A. Babar, and P. Tell, "Identifying relevant studies in software engineering," Inf Softw Technol, 2011.

[34] K. Wang, "Logistics 4.0 solution-new challenges and opportunities," in IWAMA, 2016.

[35] N. Subramanian, A. Chaudhuri, and Y. Kayıkc1, "Blockchain applications and future opportunities in transportation," in Blockchain and Supply Chain Logistics, pp. 39-48, Springer, 2020.

[36] E. Irannezhad, "Is blockchain a solution for logistics and freight transportation problems?," Transportation Research Procedia, vol. 48, pp. 290-306, 2020.

[37] S. Jagtap, F. Bader, G. Garcia-Garcia, H. Trollman, T. Fadiji, and K. Salonitis, "Food logistics 4.0: Opportunities and challenges," Logistics, vol. 5, 2021.

[38] M. S. Islam et al., "A literature review on environmental concerns in logistics: Trends and future challenges," Int. J. Logist. Res. Appl., 2021.

[39] H. Brdulak and A. Brdulak, "Challenges and threats faced in 2020 by international logistics companies operating on the polish market," Sustainability, 2021.

[40] J.-W. Han et al., "A comprehensive review of cold chain logistics for fresh agricultural products: Current status, challenges, and future trends," Trends Food Sci Technol, 2021.

[41] C. Loklindt, M.-P. Moeller, and A. Kinra, "How blockchain could be implemented for exchanging documentation in the shipping industry," in LDIC, 2018.

[42] B. Karakostas, T. Katsoulakos, and Y. Zorgios, "Towards an ict platform for the european freight transport community," IJAL, 2012.

[43] W. Hofman et al., "The applicability of blockchain technology in the mobility and logistics domain," in Towards User-Centric Transport in Europe, 2019.

[44] N. Hackius, S. Reimers, and W. Kersten, "The privacy barrier for blockchain in logistics: First lessons from the port of hamburg," in Logistics Management, 2019.

[45] U. Clausen et al., "Logistics trends, challenges, and needs for further research and innovation," in Sustainable Logistics and Supply Chains, 2016.

[46] J. Pries-Heje, R. Baskerville, and J. R. Venable, "Strategies for design science research evaluation," 2008.

[47] S. Alacam and A. Sencer, "Using blockchain technology to foster collaboration among shippers and carriers in the trucking industry: A design science research approach," Logistics, vol. 5, no. 2, 2021. 